\title{
CARDIAC DISEASES IN DOGS
}

\author{
M. HOQUE*, A. C. SAXENA, REETU ${ }^{1}$ \\ M. B. GUGJOO ${ }^{2}$ AND D. BODH ${ }^{3}$ \\ Division of Surgery \\ ICAR-Indian Veterinary Research Institute \\ Izatnagar-243 122, Uttar Pradesh, India
}

\begin{abstract}
Heart diseases are almost as common in dogs as in human subject. The clinical signs of heart disease depend on the type of disease and severity, but initially it is asymptomatic in most cases. Auscultation, electrocardiography, echocardiography and some biochemical tests are most useful diagnostic procedures employed in recognizing the cardiac diseases in dogs. Presently access to echocardiographic facility in veterinary practice has brought about a revolution in the early and precise diagnosis and management of cardiac diseases in dogs. Some congenital cardiac defects can be repaired or corrected with surgery, while other conditions can be managed with medical therapy using one or a combination of drugs. Quality of life and life expectancy can be improved with early diagnosis and treatment in congestive heart failure cases. Even though most forms cannot be prevented, heart disease caused by heartworms can easily be avoided with preventative medical therapy. With the advent of modern diagnostic modalities and increased awareness among pet owners, cardiac diseases in dogs are being attended in clinical practice.
\end{abstract}

Key words: Cardiac biomarker, Cardiac disease, Dogs, ECG, Echocardiography, Radiography

Heart disease in dogs is almost as common as it is in humans. Cardiac diseases in canines are an extensively studied phenomenon all over the world but meager information has been reported in India. The recognition of canine cardiac diseases has been delayed, and ignored on account of lack of awareness and knowledge by the owner and inadequate diagnostic facility in our country (Devi et al., 2009). Despite these numbers, many people are unaware that their dog may be at risk of heart disease. It is important that every dog considered vulnerable to cardiac diseases should be examined for cardiac function during routine examination (Vengsarkar,

\footnotetext{
*Corresponding Author

${ }^{1}$ Ranchi Veterinary College, Ranchi, Kanke

${ }^{2}$ SKUAST-K, Srinagar

${ }^{3}$ GBPUAT, Pantnagar
} 
1988). A delay in diagnosis and treatment could result in a grave prognosis or life threatening condition. Many a times the condition is detected too late until pronounced signs appear (Martin et al., 2009). The substantial involvement of heart has provoked numerous investigations leading to advancements in medical and surgical management of cardiovascular disorders. Presently access to echocardiographic facility in veterinary practice has revolutionized the field of cardiology by aiding the diagnosis and management of cardiac diseases in dogs.

\section{Incidence}

It is estimated that approximately $10-15 \%$ of all dogs are affected with heart diseases (Rush, 2002; MacPete, 2018). Heart disease in dogs may be categorized into congenital and acquired. Congenital heart disease accounts for only about $5 \%$ of all canine heart disease and is generally diagnosed when the dog is very young. Congenital cardiovascular lesions in descending order of frequency of occurrence are patent ductus arteriosus (PDA), pulmonary stenosis, aortic stenosis, persistent right aortic arch, ventricular septal defect (VSD), Tetralogy of Fallot (ToF), atrial septal defect (ASD), persistent left cranial vena cava and mitral insufficiency. Only about two-third of puppies born survives up to weaning age. Congenital cardiovascular disease has not been studied extensively (Tilley et al., 2008). The vast majority, $95 \%$, of heart disease cases are acquired. Acquired heart diseases include those that a dog naturally acquires during lifetime, usually as a result of normal wear and tear, infection or injury (Drake Centre for Veterinary Care, 2019).The most common form of heart disease in dogs is valvular disease, which primarily affects small breed dogs over 5 years of age and makes up 70$75 \%$ of heart diseases. Heartworm disease causes $13 \%$ of heart disease even though it is entirely preventable (MacPete, 2018). Myocardial disease, such as dilated cardiomyopathy (DCM), makes up $8 \%$ of heart disease and primarily affects large breed dogs of all ages (MacPete, 2018). The incidence of heart disease increases to $60 \%$ or more for dogs over 7 years of age. Ninety five per cent of heart diseases in canine are acquired, of which $75 \%$ are valvular diseases (atrioventricular valvular insufficiency) and DCM (Rush, 2002; Martin, et al., 2009). In a study most of the clinical cases of cardiac diseases were presented with a history of nocturnal coughing $(2.55 \%)$, exercise intolerance $(1.82 \%)$, partial or complete anorexia $(1.82 \%)$, swelling in abdominal area $(1.45 \%)$, dullness and depression $(0.72 \%)$, cachexia and jugular pulsation $(0.36 \%$ each) at times (Devi et al., 2009). Retrospective study revealed highest incidence of cardiac disease in dogs was of systolic dysfunction followed by dilated cardiomyopathy and congestive heart failure. Maximum incidence was seen in Labrador retriever and in male dogs. Geriatric dogs were found to be mostly affected (Reetu et al., 2017). Any type of disturbance or variance of a normal heart 
rate or rhythm in dogs is considered canine arrhythmia. In a number of cases, irregular heartbeat is caused by a hidden disorder like abnormal electrolytes, systemic disease, heart disease, injuries, or infection. An extensive study on the prevalence of arrhythmias in dogs involving 1189 referral cases for cardiological examination by electrocardiography showed $52.46 \%$ electrocardiograms with no signs of arrhythmia, but $7.99 \%$ and $39.55 \%$ pointed to physiological and pathological arrhythmias respectively. The most commonly diagnosed type was atrial fibrillation with $33.68 \%$ incidence, followed by ventricular arrhythmias (28\%), sinus pauses $(27.58 \%)$, supraventricular arrhythmias $(24 \%)$ and atrioventricular blocks $(22.95 \%)$. Pathological arrhythmias were most commonly found in male dogs and in German Shepherds (NoszczylNowak, et al., 2017).

\section{Etiopathology}

Obesity and heart diseases in dogs are encountered frequently in clinical practice. Improper feeding, inadequate exercise and managemental negligence favor obesity that may leads to heart diseases. A variety of nutritional deficiencies- dietary protein, fat, vitamins, minerals and trace elements are known to cause cardiac disease in various species (Devi and Jani, 2009). High fat diet induces abdominal obesity in dogs and results in significantly high blood pressure and heart rates, thus may lead to heart disease due to atrial hypertension and left ventricular hypertrophy. Congestive heart failure (CHF) occur most often secondary to degenerative mitral valve disease (MVD) and DCM (MacDonald et al., 2003; Serres et al., 2007; Oyama et al., 2008; Schober et al., 2010). Chronic MVD is the most common cause of heart failure in dogs which has a slow progression and rarely shows any overt clinical sign of heart disease before death (Borgarelli et al., 2008; Schober et al., 2010). Myxomatous valve disease, also known as chronic mitral valve insufficiency (CMVI) caused by progressive myxomatous degeneration of either the mitral valve alone or together with the tricuspid valve leading to incomplete coaptation of the leaflets and valvular regurgitation, is the most common heart disease which accounts for $75-80 \%$ of cardiac diseases in dogs (Pedersen et al., 1996; Haggstrom et al., 2005; Borgarelli et al., 2008). Large breed dogs are less prone to the disease than small breeds and disease tend to be age dependent as the prevalence increase dramatically in 4-5 yrs and above (Buchanan, 1977; Thrusfield et al., 1985; Borgarelli et al., 2004). Early recognition of CHF is of clinical importance in order to save the animal's life (MacDonald et al., 2003; Schober et al., 2010). There is a remarkable role of diastolic dysfunction in pathophysiology of cardiovascular diseases. The symptoms and prognosis of diastolic dysfunction which usually give rise to systolic dysfunction, determine the outcome of diseases process (Cavalcanti et al., 2007). An increased left ventricular filling pressure is indicative of left-sided congestive heart failure (Dabbah 
et al., 2006). In pericardial effusion an abnormally large amount of fluid collects in the pericardial sac with pressure elevation inside the heart leading to a lower cardiac output and eventually right-sided congestive heart failure (Gugjoo et al., 2013c; Bodh et al., 2014).

\section{Diagnosis}

Various diagnostic tools for the diagnosis of heart disease are available that include: Clinical examination and auscultation, thoracic radiography, electrocardiography, echocardiography and cardiac biomarkers (Nakayama et al., 2001). Auscultation, electrocardiography and roentgenography were the three most useful diagnostic procedures employed in recognizing the heart diseases in dogs (Detweiler et al., 1961).

The clinical signs of heart disease depend on the type of disease and severity and initially it is asymptomatic. As heart disease progresses to congestive heart failure, which occurs when the heart is unable to meet the circulatory demand, a dog may develop more obvious symptoms such as fatigue, reduced willingness to walk or exercise, difficulty breathing, loss of appetite, weight loss, a distended abdomen, trouble sleeping or coughing (MacPete, 2018). Auscultation is most helpful part of cardiac examination to detect abnormal rhythms and cardiac sound.

Thoracic radiography:Thoracic radiography is most common diagnostic tool used for cardiac diseases (Thrall, 2007). Change in cardiac size is an important guide to cardiac disease and can be used to monitor the disease and its severity (Buchanan and Bucheler, 1995; Hamlin, 1999). Cardiac size is usually assessed by comparing the appearance of the cardiac silhouette in a patient with examples of normal; however, it is very difficult to make accurate subjective assessment whether the cardiac silhouette is enlarged or misshapen (Kittleson, 1998; Lamb and Boswood, 2002). When evaluating the heart, it may be better to compare the patient's radiographs with those of a normal dog of the same breed (Lamb and Boswood, 2002). Bodh et al. (2016b) and Gugjoo et al. (2013 a, b) used vertebral scale system to measure heart size in dogs in thoracic radiograph. Objective methods for evaluating the cardiac silhouette involve measuring various cardiac dimensions and cardiothoracic ratios (Hamlin, 1968; Lamb and Boswood, 2002). The vertebral heart scale (VHS) is a method of cardiac measurement that compares the dimensions of the cardiac silhouette with the length of thoracic vertebral bodies (Buchanan and Bucheler, 1995). The generic normal range of VHS for dogs is 8.7 to 10.7 (Lamb and Boswood, 2002). VHS measurements tend to increase in dogs with cardiac disease (Nakayama et al., 2001; Lamb et al., 2001) thus, give an idea about ongoing disease process. There is a fair correlation between VHS measurements and a variety of other indices of cardiac chamber enlargement, including 
end-systolic and end-diastolic ventricular diameters as well as duration of the $\mathrm{P}$ wave and QRS complex. Lamb et al. (2001) measured vertebral heart scale on right lateral recumbent thoracic radiographs of 320 dogs of six breeds; including for each breed at least 20 dogs with no clinical signs of cardiovascular or respiratory disease and at least 19 dogs with cardiac or respiratory disease. They found significant differences between the mean values of the scale for the different breeds. For all the breeds except one, they recorded a trend for dogs with cardiac disease (but not respiratory disease) to have higher mean values on the scale than normal dogs of the same breed; however, at the optimal value of the scale for distinguishing between dogs of each breed with and without cardiac disease, the sensitivity and specificity were relatively low, in the range 58 to 83 per cent. Lamb et al. (2001) assessed the influence of VHS on the accuracy of the radiographic diagnosis of cardiac disease. They examined thoracic radiographs of $50 \mathrm{dogs}$ with proven cardiac disease, 26 with other thoracic diseases, and 50 with no clinical signs of cardiovascular or respiratory disease with the help of three independent, blinded observers and recorded a VHS over 10.7 on the lateral radiograph as moderately accurate sign of cardiac disease. Similarly, Greco et al. (2008) assessed the effect of right vs. left recumbency on computation of the vertebral heart score (VHS) in 63 healthy dogs and found a signiûcantly higher VHS in right lateral recumbency $(9.8 \pm 0.6$ vs. $9.5 \pm 0.8 ; \mathrm{P}<0.0004)$. They also recorded more difference between left and right side measurements when considering the type of thorax while there was no significant influence of gender and dog size on VHS values. Grant et al. (2013) described VHS measurements in eight dog breeds viz. Pug, Pomeranian, Yorkshire Terrier, Dachshund, Bulldog, Shih Tzu, Lhasa Apso and Boston Terrier. They found significantly greater VHS $(>9.7 \pm 0.5)$ in Pug, Pomeranian, Bulldog and Boston Terrier groups and a significant effect of body condition score on the VHS of Lhasa Apso group. Anomalous vertebrae in the thoracic column were associated with a significant increase in VHS of the Bulldog $(\mathrm{P}=0.028)$ and Boston terrier $(\mathrm{P}=0.0004)$ groups. But no significant effect on thoracic depth to width ratio on VHS was seen. Measuring the cardiac silhouette does not aid diagnosis of cardiac disease because there is considerable overlap in results from dogs with cardiac disease and normal dogs. This overlap occurs partly because dogs with concentric hypertrophy and those examined in the early stages of their disease may not have any significant cardiac enlargement and partly because certain breeds have relatively large appearing hearts (Lamb and Boswood, 2002). There is also evidence that females have smaller mean VHS measurements than males (Lamb et al., 2001). Even when using breed-specific normal VHS ranges, there is still significant overlap between normal dogs and dogs with cardiac disease. At the optimal VHS value for separation of cardiac from non cardiac diseased dogs of each 
breed, the accuracy is relatively low (range, $58 \%$ to $83 \%$ ) (Lamb et al., 2001; Lamb and Boswood, 2002). The limitations support the need for other diagnostic modality for the confirmatory diagnosis of cardiac diseases in dogs. Although reliability of clinical signs is limited still CHF can be suspected on the basis of clinical signs. Thoracic radiography is the most commonly applied method for the diagnosis of $\mathrm{CHF}$ and is considered the clinical "gold standard' (Balbarini et al., 1991). However, sensitivity and specificity of radiograph is doubtful, especially in the cases of combined heart and lung disease.

\section{Electrocardiography:}

The electrocardiogram (ECG) is the voltage time graph of the electrical activities of the heart and widely used as a powerful noninvasive diagnostic tool for monitoring heart rate (HR), cardiac rhythm, conduction integrity and electrical axis (Mukherjee et al., 2015) and in detecting cardiac abnormalities in dogs (Tilley, 1985). In dogs, cardiac arrhythmias and intra-cardiac conduction disturbances are common problems, which can be well elucidated using ECG (Gugjoo et al., 2014a). In 1949 Lannek reported a detailed statistical analysis of the normal electrocardiogram of normal dog, together with observation on a series of animal showing electrocardiogram abnormalities. Bohn et al. (1971) used electrocardiography to study the influence of age, sex and breed on atrial fibrillation in dogs, its association with various types of underlying heart disease and its prognostic significance. Taylor and Sittnikow (1968) used auscultation, roentgenograms and electrocardiograms in diagnosing cardiac diseases in dogs, and found that reported careful auscultation was the most important method of diagnosing cardiac disease but confirmation of a diagnosis of cardiac disease and evaluation of its severity can be best obtained through the use of the roentgenogram and electrocardiogram. Dhanapalan et al. (1997) reported electrocardiographic observation in Spitz, Doberman, Labrador, German Shepherd and Mongrels. They observed breed variation with respect to $P$ wave and $\mathrm{R}$ wave amplitudes without much variation in QRS complex. Pereira et al. (2004) reported that increase in the amplitude of $\mathrm{R}$ wave, QRS duration and deep Q wave in Lead II ECG constituted a good indicator of cardiomyopathy in Cocker Spaniel.

The axis of the ECG is the major direction of the overall electrical activity of the heart and an important indicator to determine cardiac diseases. The normal adult QRS axis is between -30 degrees and +90 degrees, which is directed downward and to the left. If the QRS axis falls between -30 degrees and -90 degrees, it is considered left axis deviation (LAD). In this case, the QRS vector is directed upward and to the left. If the QRS axis falls between +90 degrees and 180 degrees or beyond +100 degrees if the adult range is used, then right axis deviation (RAD) is present. The QRS vector would be directed downward 
and to the right. If the QRS axis happens to fall between -90 degrees and 180 degrees, this would be referred to as extreme axis deviation, whereby the ventricular vector is directed upward and to the right. Lastly, if the QRS complex is isoelectric or equiphasic in all leads with no dominant QRS deflection, it is considered indeterminate axis. The causes of LAD include: normal variation (physiologic, often age-related change), left ventricular hypertrophy, conduction defects: left bundle branch block, left anterior fascicular block. On the other hand RAD indicates to an exacerbation of lung disease, a pulmonary embolus, a new high lateral MI (Qr pattern) or simply a tachycardia (Kashou and Kashou, 2018).

Ambulatory electrocardiography (Holter monitoring and cardiac event recording) is also used for diagnosis of cardiac disease (Tilley et al., 2008).

Cardiac catheterization-The standard CC procedure for evaluation of congenital and acquired cardiac diseases typically include measurement of intra cardiac pressure, blood oximetry and selective angiocardiography. The most common indication for $\mathrm{CC}$ is to ameliorate congenital heart diseases (e.g. pulmonary stenosis or PDA). For the purpose of diagnosis, advances in echocardiography have markedly reduced the need for routine CC (Tilley et al., 2008).

Echocardiography: Echocardiography has emerged as the most versatile imaging technique for the study of cardiac diseases (Muzzi et al., 2006; Gugjoo et al., 2014b,c,d; Bodh et al., 2016a; Reetu, 2018). A unidimensional view is obtained through M mode echocardiography which provide image of heart visualized over various cardiac cycle (Cornell et al., 2004). Usually it is used to measure linear cardiac dimensions of cardiac chambers walls and great vessels. A significant correlation has been seen between body size and aortic, left atrial, left ventricular, septal and posterior wall dimensions, and mitral wall amplitude of motion (Lombard, 1984; Jacobs and Mahjoob, 1988; Della et al., 2000; Muzzi et al., 2006). Body surface area is much more closely related to echocardiographic measurements than body size and confirmation (Della et al., 2000). Crippa et al. (1992) measured echocardiographic parameters and indices in normal Beagle dogs and found that the morphofunctional cardiac homogeneity is independent of size and age in Beagles aged around 28 weeks. Boon (2011) evaluated twenty young healthy dogs weighing from 9.8 to $28.6 \mathrm{~kg}$ by M-mode echocardiography and found statistically significant correlation to body size for the aortic, left atrial, left ventricular, septal, and posterior wall dimensions and the mitral valve amplitude of motion. Echocardiography has been used for standardization of cardiac indices and for evaluation of cardiac function in healthy dogs of different breeds viz., Labrador retriever, Indian Spitz (Gugjoo, 2011; Saxena, 2008; Bodh, 2014). Snyder et al. 
(2005) evaluated healthy, non exercising, awake Greyhounds to reveal substantial differences in left ventricular cavity dimensions, wall thickness, systolic time intervals and fractional shortening as compared to previously reported normal echocardiographic values obtained from Mongrels and various other dog breeds. Echocardiography is useful in the evaluation of patient with congenital heart disease like obstruction of the ventricular outflow, PDA, VSD, ASD, ToF, dysplasia of the mitral and tricuspid valves and acquired heart disease like valvular disease, congestive (dilated) cardiomyopathy (CCM), hypertrophic cardiomyopathy (HCM), pericardial effusion etc. (Bonagura, 1983; Gugjoo et al., 2013c; Bodh et al., 2014; Bodh et al., 2016a). It can be valuable for evaluating dogs with naturally occurring pericardial disease. DCM can be diagnosed through echocardiographic reference values as the values significantly vary between the diseased and normal dogs (Koch et al., 1996). Rao et al. (2008) used M-mode echocardiogram for measurement of echocardiographic indices and found it a useful technique in recognizing left ventricular dilation and poor contractile function in dogs with DCM. Evaluation of left ventricular diastolic function provide valuable information to determine severity and prognosis of cardiac disease (Hori et al., 2008). M- mode echocardiography is useful for early and confirmatory diagnosis and to quantify the left ventricular dysfunction in dogs with dilated cardiomyopathy (Sleeper et al., 2002; Rao et al., 2008). De Madron et al. (2011) reported baseline echocardiographic data in dogs with congestive heart failure caused by mitral valve disease. Gugjoo et al. $(2014 c, d)$ reviewed various cardiac diseases and normal reference values of $\mathrm{M}$ - mode echocardiograph in different dog breeds and found it helpful diagnostic technique when employed with other diagnostic modalities. Two-dimensional real-time ultrasonography (2D) is a two dimensional tomographic image of the various cardiac structures. This mode can be used to recognize lesions such as valvular abnormalities, heart size, pericardial effusion and cardiac tumors. Thomas et al. (1993) recommended standardized imaging planes and display conventions for twodimensional echocardiography in dogs and cats. He recommended three transducer locations ("windows") to access consistent imaging planes: the right parasternal location, the left caudal (apical) parasternal location and the left cranial parasternal location. Rishniw and Erb (2000) evaluated four 2-dimensional echocardiographic methods of assessing left atrial size in dogs. They measured left atrium at specific time points in the cardiac cycle and found strong associations of LA dimensions with body weight. They also provided echocardiographic data with reference intervals for 2D echocardiographic estimates of LA size in adult dogs.

Doppler Echocardiography (DE): Pulse wave Doppler (PW-D), continuous wave Doppler (CW-D) and color Doppler (CD) 
are the types of DE. DE provides valuable information regarding hemodynamic of heart so as any disorder can be diagnosed before occurrence of any irreversible structural and functional deformity. It can identify the nature and the severity of cardiac disease. CD is used to detect the direction of flow of blood, PW-D determines the velocity of blood flow and CW-D is used for more turbulent flow. Before the introduction of PW-D, echocardiography using mitral leaflet motion analysis was used to demonstrate impaired early ventricular filling (Leischik et al., 2015). DE has major advantages over currently used invasive methods of obtaining physiological information with respect to intracardiac hemodynamic. PWD was validated using invasive measurements (Appleton et al., 1994), is now the most widely used technique to directly analyze diastolic function and indirectly analyze atrial function (Reetu, 2018). The pressure gradient across a stenotic valve can be calculated from the measurement by the application of Bernouli's principle (Berger et al., 1984). Mitral and pulmonary flow velocities reliably correlate to mean LA pressures and to pressure changes (Appleton et al., 1994; Leischik et al., 2015). The mitral inflow velocity profile is determined in a complex manner by multiple factors, which include left atrial pressure, relaxation, LV systolic pressure, ventricular suction, preload, heart rate and atrial function (Choong et al., 1988; Schober et al., 2010). The pattern of LV filling determined by Doppler transmitral flow is used to noninvasively evaluate LV diastolic performance and has been described in detail in people and validated in experimental dogs (Choong et al., 1988). With the simultaneous use of hemodynamic and Doppler techniques Appleton, (1991) studied mitral flow velocity, mitral valve motion and transmitral pressure gradient during 50 cardiac cycles each of spontaneous or atrial paced first and second-degree atrioventricular block in five lightly sedated dogs. They found Diastolic mitral regurgitation as a common finding that can he detected with use of DE techniques in patients with conduction abnormalities. DE is helpful in diagnosing mitral valve degeneration and DCM, and can be used to predict chronic heart failure secondary to them in dogs (Schober et al., 2010). The major finding is that it allow for a rapid and feasible estimation of whether or not $\mathrm{CHF}$ is present. In addition, respiration rate taken at physical exam was equally useful in the prediction of CHF (Schober et al., 2010). The diagnosis of CHF in dogs with degenerative mitral valve disease relies on a combined interpretation of clinical signs and results of more than one diagnostic tools including thoracic radiography, NT proBNP or DE (Chetboul et al., 2009). Measurements of cardiac output and regurgitant volumes, quatitation of intracardiac shunts and evaluation of systolic and diastolic function of heart can be done with DE (Boon, 2011). Diastolic dysfunction contributes significantly to the pathophysiology and symptomatology of a 
large number of cardiovascular disorders but early stages of LV dysfunction most often seen in asymptomatic dogs with heart disease (or healthy dogs older than 10 years) (Schober and Fuentes, 2001). Chetboul and Tissier (2003) discussed the accuracy of conventional echo-Doppler examination in diagnosis of canine MVD and of its consequences on cardiac remodeling, LV function and cardiovascular pressures. They recommended serial echo Doppler examinations in dogs with MVD in order to identify and track these alterations over time and detect an ongoing worsening of the disease, they also suggested several standard echo Doppler variables for asymptomatic dogs mainly include grading of MR severity, evaluation of pulmonary arterial pressures, assessment of filling pressures and left heart changes (LA/Ao, FS\%, LV diameters, LV volumes and corresponding ESVI and EF\%) using a planimetric method that may help in predicting animals at risk for decompensation thus, they can allow an early detection of dogs at risk for rapid progression into congestive heart failure. The degree of mitral regurgitation is the key factor to decide left atrial size and severity of disease in dogs with mitrial regurgitation (Kittleson and Brown, 2012). Mitral regurgitation might be detected and quantified by spectral or colour flow Doppler ultrasound. The regurgitant flow should be aligned to ultrasound beam and this was most often achieved in the apical four chamber view. Spectral Doppler mapping can be used to identify the regurgitant jet while color Doppler mapping is not available (Kittleson, 1998). However systolic dysfunction is not common in small breed dogs, it can be seen in end stages of chronic mitral valve insufficiency and myocardial infarcts where contractile performance may be diminished with the effect of valve insufficiency (Borgarelli et al., 2007). Clinical diagnosis of pulmonary hypertension in dogs typically relies on Doppler echocardiographic estimates of pulmonary arterial pressure derived from tricuspid or pulmonary regurgitant jets (Visser et al., 2016). Pulmonary hypertension in dogs can be primary or secondary to various diseases, including pulmonary venous and left atrial hypertension (e.g., degenerative mitral valve disease and left-sided congestive heart failure), pulmonary thromboembolic disease (e.g., dirofilariasis, Cushing's syndrome, protein losing nephropathy, or enteropathy), congenital cardiac abnormalities (e.g., reversed PDA), and chronic pulmonary disease (e.g., chronic bronchitis or pulmonary fibrosis) (Bach et al., 2006; Johnson, 1996). The underlying causes of pulmonary hypertension are not readily reversible, and prognosis of severe pulmonary hypertension is poor in both human and veterinary medicine (Johnson et al., 1999; Kellum and Stepien, 2007). Therefore, early recognition of the pulmonary hypertension is essential in veterinary practice. Non invasive prediction of PAP is possible with Doppler echocardiography using the modified 
Bernoulli equation applied to the peak velocity of tricuspid regurgitation or pulmonary regurtitant flow (Lee et al., 2015). Doppler derived velocities and pressure gradient were recorded in healthy Spitz and Labrador retriever dogs of different age groups as reference data for diagnosis of various cardiac affections in dogs (Reetu, 2018).

Cardiac biomarker: A cardiac biomarker is a parameter that can predict or diagnose a cardiac disease either if it is primary or secondary with as high sensitivity and specificity as possible. The reason of the increasing interest in cardiac biomarkers is due to its convenience, low cost, noninvasiveness, risk free, fast turn-around time, efficient and highly predictive tests. In the last 30 years many researchers have studied various types of cardiovascular markers, aiming to define better diagnostic aspects of primary and secondary cardiac diseases. In the veterinary field, the most complete studies have been performed in small animals, mainly dogs and cats. At present, in veterinary clinical practice, the biomarkers with the highest predictibility for cardiac diseases are N-terminal probrain natriuretic peptide (NT-pro BNP) followed by cardiac troponins I and T (cTnI and cTnT), and less frequent used, atrialnatriuretic peptide, endothelin, tumor necrosis factor $\alpha$ (TNF $\alpha$ ) and C-reaction protein, that show lesser specificity (Fonfara et al., 2010; Saunders et al., 2009). The structure, metabolism, function, applicability, reference values and variations in different diseases of troponin I and N-terminal proBNP in dogs and cats have been extensively reviewed (Baisan et al., 2016). Concentration of certain enzymes and isoforms also depict the ongoing degenerative change hence it can be used to support the diagnosis of cardiac diseases. Creatine kinase concentration in serum or plasma elevate immediately after any damage to muscle or myocardium. Shell et al. (1971) assessed the extent of myocardial infarction in the conscious dog by analyzing serial changes in serum creatine phosphokinase activity and found the method suitable for assessment of infarct size over a wide range and for detection of modification of infarct size produced by pharmacologic and physiologic interventions. Hashimoto et al. (1985) used plasma MM creatine kinase (CK) isoforms concentration for early detection of myocardial infarction in eight conscious dogs and found immediate increases in concentration of CKMM isoform on response to myocardial infarction. They conclude that initial CK release indicative of infarction is detectable within $1 \mathrm{hr}$ after the onset of ischemia by quantification of plasma CKMM isoforms. Creatine kinase $(\mathrm{CK})$ and its fraction $\mathrm{CK}$ $\mathrm{MB}$ are also biomarkers used to assess the cardiac function in different types of pathologies or surgery techniques developing myocardial damage, such as ischemia, myocardial necrosis or percutaneous coronary intervention in humans (Apak et al., 2005). This biomarker is not specific to myocardial tissue, being 
released also during non-cardiac muscle damage. Apak et al. (2005) concluded that cardiac troponin $\mathrm{T}$ is a more specific and sensitive marker for myocardial injury than CK-MB. Despite this, the biomarkers are not stand-alone tests and their results should be evaluated in the context of the medical history, physical examination and other diagnostic tests. The biomarkers discussed in this paper are the most used for assessing cardiac condition, with diagnostic potential in most pathologies, but reliability is still lacking and further researches are required (Baisan et al., 2016).

\section{Treatment}

Treatment should be specific for the type of disease. Some congenital cardiac defects can be repaired or corrected with surgery, while other conditions can be managed with medical therapy using one or a combination of drugs. Cardiac surgery is increasingly an option for management of congenital and acquired cardiac conditions in dogs. Some cardiac surgeries are widely available, whereas open cardiac repairs that require cardiopulmonary bypass (CPB) are currently only performed in a few specialized centers. Some cardiac surgeries are performed with curative intent, whereas others are considered palliative only. Cardiac surgeries included closed cardiac surgeries, cardiac surgeries performed during inflow occlusion and cardiac surgeries performed under CPB. Pacemaker therapy has become a common method of treating symptomatic bradycardia in dogs (Tilley et al., 2008). In dogs with congestive heart failure, a low-sodium diet may be recommended to help eliminate excess fluid in the body. In general, the goals of treatment are to minimize damage to the heart muscle, control the accumulation of fluids in the lungs, improve circulation, regulate the heart rate and rhythm, ensure that there is enough oxygen in the blood, and minimize the risk of blood clot formation. In heartworm disease, the mature heartworms and larvae should be killed. Ultimately, the goals are achieved when treatment resolves the signs, the breathing and heart rates are normal at rest, and the dog has a good quality of life.

Common drugs used: There are many medications for dogs with cardiovascular disease. Diuretic (furosemide) is a mainstay of therapy in dogs with congestive heart failure $(\mathrm{CHF})$ due to degenerative mitral valve disease (DMVD) or dilated cardiomyopathy (DCM). It increases urine output and is important and effective means for removing fluids that accumulate in dogs with heart problems. The use of a diuretic can be life saving when a dog is in a crisis caused by heart failure. Some drugs, such as the digitalis glycoside called digoxin, increase the force of heart muscle contraction, slow the heart rate, and allow blood pressure receptors to work better. They are used to treat heart failure. Severe heart disease is generally associated with activation of the renin-angiotensinaldosterone system (RAAS), which promotes fluid retention, vasoconstriction, and myocardial and vascular remodeling. 
Use of angiotensin-converting enzyme (ACE) inhibitors (enalapril, benazepril, ramapril, and lisinopril) in dogs with $\mathrm{CHF}$ is associated with improved quality of life and survival. They can reduce blood pressure, improve cardiac output, and reduce the backward flow of blood caused by a leaky heart valve (Oyama, 2009). Pimobendan is a positive inotrope and increases contractility through a mechanism different from that of traditional inotropes such as digoxin, the advantage of which is increased contractility without significant increases in myocardial oxygen demand. It relaxes vascular smooth muscle and elicits modest arterial vasodilation; this dual "inodilating" action is unique. It also improves survival and quality of life in dogs with DMVD, and very likely does the same in dogs with DCM (Boswood, 2010). Sildenafil is a medication originally discovered in 1989 by Pfizer used to treat heart related chest pain and pulmonary arterial hypertension as it dilates arteries leading to the lungs. It is also used to treat erectile dysfunction in man. Other types of drugs can strengthen respiratory muscles and improve breathing. Some medications are prescribed to control arrhythmias, slow or speed heart rate, and prevent blood clots. Treatment of cardiac arrhythmia is included: Sinus arrhythmia is usually treated with medication such as Atropine or Mexiletine. Atrial fibrillation is regulated by Digoxin and possibly extended-release Diltiazem. Atrioventricular block can be treated by Theophylline or Propantheline. In some cases, a pacemaker needed to be placed surgically. Ventricular tachycardia (VT) may be treated with antiarrhythmic therapy in breeds predisposed to this condition. Lidocaine, Sotalol and Mexiletine are sometimes given if the dog is in acute VT. Premature beat is medicated with Mexiletine or Sotalol (Cunningham and Roderick, 2019). In other cases, medications are provided to kill heartworms or other parasites in the cardiovascular system. Nutritional management of dogs with cardiac problems can be handled by providing adequate calories, protein and modulating cytokine production to manage the possible cause of decreased appetite of the side effects of medications. Specifically, coenzyme Q10 (CoQ10), vitamin E (as alpha-tocopherol), L-carnitine, taurine, and fish oil (omega-3 fatty acids) have all been evaluated in the prevention and treatment of many types of heart disease in dogs. Other supplements with preliminary evidence, meriting further investigation, include magnesium and the $\mathrm{B}$ vitamins. Both clinical observation and interventional trials with various breeds have provided clear evidence for the benefit of numerous supplements on canine heart disease. Appropriate levels of certain dietary nutrients have been shown to increase life span, improve life quality, reduce symptoms and physical evidence of disease, and decrease mortality rates in these animals (Dove, 2001). Stem cell therapy 
in cardiac diseases is coming up as new area (Saxena, 2014). A study with multiple intravenous injections of puppy deciduous teeth stem cells (pDSCs) suggested that pDSCs could be a supplement for valvular heart disease treatment in dogs (Petchdee and Sompeewong, 2016). The full potential

\section{REFERENCES}

Apak I, Iltumur K, Tamam Y and Kaya N, 2005. Serum cardiac troponin $\mathrm{T}$ levels as an indicator of myocardial injury in ischemic and hemorrhagic stroke patients. Tohoku J Exp Med, 205: 93-101

Appleton CP, 1991. Influence of incremental changes in heart rate on mitral flow velocity: Assessment in lightly sedated, conscious dogs. J Am Coll Cardiol, 17(1): 227-236

Appleton CP, Gonzalez MS and Basnight MA, 1994. Relationship of left atrial pressure and pulmonary venous flow velocities: Importance of baseline mitral and pulmonary venous flow velocity patterns studied in lightly sedated dogs. J Am Soc Echocardiogr, 7(3): 264-275

Bach JF, Rozanski EA, MacGregor J, Betkowski JM and Rush JE, 2006. Retrospective evaluation of sildenafil citrate as a therapy for pulmonary hypertension in dogs. J Vet Intern Med, 20(5): 1132-1135

Baisan RA, Rosa AD, Loria AD, Vulpe V and Pinantedosi D, 2016. Cardiac biomarkers in clinical practice in dogs and cat- A review. Human Vet Med, 6(1)51-58 of stem cell therapy to treat heart disease in dog is yet to establish (Rajewski, 2017). Gene therapy in cardiovascular disease is the forefront of new therapeutic options, and with additional development and rigorous testing, could become the paradigm for first round treatment options in the clinic (Wolfram and Donahue, 2013).

Balbarini A, Limbruno U, Bertoli D, Tartarini G, Baglini R et al., 1991. Evaluation of pulmonary vascular pressures in cardiac patients: The role of the chest roentgenogram. J Thorac Imaging, 6: 6268

Boswood A, 2010. Current use of pimobendan in canine patients with heart disease. Vet Clin North Am Small Anim Pract, 40(4): 571-80, doi: 10.1016/j.cvsm.2010.04.003

Berger M, Russell L, Berdoff Peter E, Gallerstein and Goldberg E, 1984. Evaluation of aortic stenosis by continuous wave Doppler ultrasound. J Am Coll Cardiol, 3(1): 150-156

Bodh D, 2014. Evaluation of cardiac function in Spitz, Labrador retriever and non descript dogs with special reference to Doppler parameters. Ph. D thesis, IVRI, Izatnagar

Bodh D, Hoque M, Saxena AC, Gugjoo MB and Pawde AM, 2014. Radiographic, electrocardiographic and echocardiographic features of occult dilated cardiomyopathy in Labrador retrievers. Indian J Vet Surg, 35 (2): 134-137

Bodh D, Hoque M, Saxena AC, Gugjoo MB and Bist D, 2016a. Diagnosis of dilated 
cardiomyopathy and pericardial effusion in two dogs. Indian J Vet Surg, 37(1): 48- 50

Bodh D, Hoque M, Saxena AC, Gugjoo MB, Bist D et al., 2016b. Vertebral scale system to measure heart size in thoracic radiographs of Indian Spittz, Labrador retriever and Mongrel dogs. Vet World, 9(4): 371-376

Bohn FK, Pallerson F and Pyle RL, 1971. Atrial fibrillation in dogs. Br Vet J, 127(10): 485496

Bonagura JD, 1983. M-Mode echocardiography- Basic principles. Vet Clin North Am Small Anim Pract, 13(2): 299-319

Boon JA, 2011. Veterinary echocardiography. $2^{\text {nd }}$ edn., Wiley- Blackwll, Chichester

Borgarelli M, Savarino P, Crosara S, Santilli RA, Chiavegato D et al., 2008. Survival characteristics and prognostic variables of dogs with mitral regurgitation attributable to myxomatous valve disease. J Vet Intern Med, 22(1): 120-128

Borgarelli M, Tarducci A, Zanatta R and Haggstrom R, 2007. Decreased systolic function and inadequate hypertrophy in large and small breed dogs with chronic mitral valve insufficiency. J Vet Intern Med, 21: 61-67

Borgarelli M, Zini E, D’Agnolo G, Tarducci A, Santilli RA et al., 2004. Comparison of primary mitral valve disease in German Shepherd dogs and in small breeds. J Vet Cardio, 6(2): 27-34

Buchanan JW, 1977. Chronic valvular disease (endocardiosis) in dogs. Adv Vet Sci Comp Med, 21: 75-106
Buchanan JW and Bucheler H, 1995. Vertebral scale system to measure canine heart in radiographs. J Am Vet Med Assoc, 206: 194-199

Cavalcanti GAA, Muzzi RAL, Araujo RB and Cherem M, 2007. Doppler echocardiographic assessment if diastolic function in Boxer dogs. Arq Bras Vet Zootec, 59(5): 1169-1176

Chetboul V and Tissier,Rm 2003. Echocardiographic assessment of canine degenerative mitral valve disease. J Vet Intern Med, 17: 84-88

Chetboul V, Serres F, Tissier R, Lefebvre HP, Carlos Sampedrano C et al., 2009. Association of plasma N Terminal Pro B type natriuretic peptide concentration with mitral regurgitation Severity and outcome in dogs with asymptomatic degenerative mitral valve disease. J Vet Intern Med, 23(5): 984-994

Choong CY, Abascal VM, Thomas JD, Guerrero JL et al., 1988. Combined influence of ventricular loading and relaxation on the transmitral flow velocity profile in dogs measured by Doppler echocardiography. Circulation, 78(3): 672683

Cornell CC, Kittleson MD, Torre, PD, Häggström J, Lombard C W et al., 2004. Allometric scaling of $\mathrm{m}$ mode cardiac measurements in normal adult dogs. J Vet Intern Med, 18(3): 311-321

Crippa L, Ferro E, Melloni E, Brambilla P and Cavalletti E, 1992. Echocardiographic parameters and indices in the normal 
Beagle dog. Lab Anim, 26: 190-195

Cunningham SM and Roderick KV, 2019.

Treatment of cardiovascular disease in dogs. www.msdvetmanual.com

Dove RS, 2001. Nutritional therapy in the treatment of heart disease in dogs. Alter Med Review, 6 (Suppl): 38-45

Dabbah S, Reisner SA, Aronson D and Agmon Y, 2006. Left ventricular filling hemodynamics in patients with pulmonary edema and preserved versus reduced left ventricular ejection fraction: A prospective Doppler echocardiographic study. J Am Soc Echocardiogr, 19: 733-743

de Madron E, Jonathan N King JN, Strehlau G and White RV, 2011. Survival and echocardiographic data in dogs with congestive heart failure caused by mitral valve disease and treated by multiple drugs: A retrospective study of 21 cases. Can Vet J, 52(11): 1219-1225

Della Torre PK, Kirby AC, Church DB and Malik R, 2000. Echocardiographic measurements in Greyhounds, Whippets and Italian Greyhounds - dogs with a similar conformation but different size. Aust Vet J, 78: 49-55

Detweiler DK, Patterson DF Hubben K and Botts RP, 1961. The prevalence of spontaneously occurring cardiovascular disease in dogs. Am J Public Hlth, 51(2): 228-241

Devi S, Jani RG, Anne FK and Singh RD, 2009. Study on clinical symptoms in canine cardiac diseases. Vet World, 2(8): 307-309

Dhanapalan P, Vijayan R and Chitra, 1997.
Electrocardiographic values among breeds of dogs. Indian J Vet Med, 17: 65-67

Drake Centre for Veterinary Care, 2019. Heart disease in dogs. www.the drakecentre.com

Fonfara S, Loureiro J, Swift S, James R, Cripps $\mathrm{P}$ et al., 2010. Cardiac troponin I as a marker for severity and prognosis of cardiac disease in dogs. Vet J, 184: 334-339

Grant KJ, Pollard RE and Johnson LR, 2013. Vertebral heart scores in eight dog breeds. Vet Radiol Ultrasound, 54(1): 3-8

Greco A, Meomartino L, Raiano V, Fatone G and Brunetti A, 2008. Effect of left vs. right recumbency on the vertebral heart score in normal dogs. Vet Radiol Ultrasound, 49(5): 454-455

Gugjoo MB, 2011. Determination of radiographic, electrocardiographic, echocardiographic and haematobiochemical cardiac reference value in Labrador retriever. M.V.Sc. thesis, IVRI, Izatnagar

Gugjoo MB, Hoque M, Saxena AC, Zama MMS and Amarpal, 2013a. Vertebral scale system to measure heart size in dogs in thoracic radiographs. Adv Anim Vet Sci, 1(1): 1-4

Gugjoo MB, Hoque M, Zama MMS, Saxena AC, Pawde AM et al., 2013b. Vertebral scale system to measure heart size on thoracic radiographs of Labrador retriever dogs. Indian Vet J, 90(2): 71-73

Gugjoo MB, Hoque M, Saxena AC, Zama MMS and Yatoo MI, 2013c. Pericardial effusion in dogs: Diagnostic features of radiography, electrocardiography and 
echocardiography. International J Current Res, 5(12): 4233-4237

Gugjoo MB, Hoque M, Saxena AC and Suz Zama MMS, 2014a. Reference values of six- limb-lead electrocardiogram in conscious Labrador retriever dogs. Pak J Biol Sci, 17(5): 689-695

Gugjoo MB, Hoque M, Saxena AC, Zama MS and Dey S, 2014b. Reference values of Mmode echocardiographic parameters and indices in conscious Labrador retriever dogs. Iran J Vet Res, 15(4): 341-346

Gugjoo MB, Saxena AC, Hoque M and Zama MMS, 2014c. M-mode echocardiographic study in dogs. Afr J Agri Res, 9(3): 387396

Gugjoo MB, Saxena AC, Hoque M, Mehenran $\mathrm{K}$ and Zama MMS. 2014d. Pericardial effusion in a Labrador retriever dog. Asian J Anim Sci, 8: 34-37

Haggstrom J, Kvart, C and Pedersen HD, 2005. Acquired valvular disease. In: Ettinger SJ, Feldman EC, edn., Textbook of Veterinary Internal Medicine, $6^{\text {th }}$ edn., Elsevier, St Louis. pp1022-1039

Hamlin RL, 1968. Analysis of the cardiac silhouette in dorsoventral radiographs from dogs with heart disease. J Am Vet Med Asso, 153(11): 1446

Hamlin RL, 1999. Pathophysiology of the failing heart. In: Fox PR, Sisson D, Moise NS (edn.): Textbook of Canine and Feline Cardiology, WB Saunders Co. Philadelphia, pp205-215

Hashimoto H, Abendschein DR, Strauss AW and Sobel BE, 1985. Early detection of myocardial infarction in conscious dogs by analysis of plasma MM creatine kinase isoforms. Circulation, 71(2): 363-369

Hori Y, Kanai K, Nakao R, Hoshi F and Higuchi SI, 2008. Assessing diastolic function with doppler echocardiography using a novel index: ratio of the transmitral early diastolic velocity to pulmonary diastolic velocity. J Vet Med Sci, 70(4): 359-366

Jacobs G and Mahjoob K, 1988. Multiple regression analysis, using body size and cardiac cycle length, inpredicting echocardiographic variables in dogs. Am J Vet Res, 49: 1290-1294

Johnson L, 1996. Diagnosis of pulmonary hypertension. Clin Tech Small Anim Pract, 14: $231-236$

Johnson L, Boon J and Orton EC, 1999. Clinical characteristics of 53 dogs with Doppler derived evidence of pulmonary hypertension: 1992-1996. J Vet Intern Med, 13(5): 440-447

Kashou AH and Kashou HE, 2018. Electrical axis (Normal, Right Axis Deviation and Left Axis Deviation). StatPearls

Kellum HB and Stepien RL, 2007. Sildenafil citrate therapy in 22 dogs with pulmonary hypertension. J Vet Intern Med, 21: 12581264

Kittleson MD and Brown WA, 2012. Regurgitant fraction measured by using the proximal isovelocity surface area method in dogs with chronic myxomatous mitral valve disease. J Vet Cardiol, 14: 127-148

Kittleson MD, 1998. Primary myocardial 
disease leading to chronic myocardial failure. Small animal cardiovascular medicine. St. Louis: Mosby, pp319-346

Koch J, Pedersen HD, Jensen AL and Flagstad A, 1996. M mode echocardiographic diagnosis of dilated cardiomyopathy in giant breed dogs. J Vet Med A, 43(1 10): 297-304

Lamb CR, Wikeley H, Boswood A and Pfeiffer DU, 2001. Use of breed-specific ranges for the vertebral heart scale as an aid to the radiographic diagnosis of cardiac disease in dogs. Vet Rec, 148(23): 707-711

Lamb CR and Boswood A, 2002. Role of survey radiography in diagnosing canine cardiac disease. Compendium on Continuing Education for the Practicing Veterinarian -North American Edition, 24(4): 316-326

Lee Y, Choi W, Lee D, Chang J, Kang JH et al., 2015. The correlation between caudal pulmonary artery diameter to body surface area ratio and echocardiography-estimated systolic pulmonary arterial pressure in dogs. J Vet Sci, 17(2): 243-251

Leischik R, Littwitz H, Dworrak B, Garg P, Zhu $\mathrm{M}$ et al., 2015. Echocardiographic evaluation of left atrial mechanics: Function, history, novel techniques, advantages, and pitfalls. Bio Med Research Internationa, pp765921

Lombard CW, 1984. Normal values of the canine M-mode echocardiogram. Am J Vet Res, 45: 2015-2018

MacDonald KA, Kittleson MD, Munro C and Kass P, 2003. Brain natriuretic peptide concentration in dogs with heart disease and congestive heart failure. J Vet Intern Med, 17(2): 172-177

MacPete, R. 2018. Dogs and Heart Disease: An Overview. IDEXX Laboratories Inc. www.pethealthnetwork.com

Martin MWS, Johnson MJS and Celona B, 2009. Canine dilated cardiomyopathy: A retrospective study of signalment, presentation and clinical ûndings in 369 cases. J Small Anim Pract, 50: 23-29

Mukherjee J, Das PK, Ghosh PR, Banerjee D, Sharma T et al., 2015. Electrocardiogram pattern of some exotic breeds of trained dogs: A variation study. Vet World, 8(11): 1317-1320

Muzzi RAL, Muzzi LAL, De Araujo RB and Cherem M, 2006. Echocardiographic indices in normal German Shepherd dogs. J Vet Sci, 7(2): 193-198

Nakayama $\mathrm{H}$, Nakayama $\mathrm{T}$ and Hamlinxya RL, 2001. Correlation of cardiac enlargement as assessed by vertebral heart size and echocardiographic and electrocardiographic findings in dogs with evolving cardiomegaly due to rapid ventricular pacing. J Vet Intern Med, 15(3): 217-221

Noszczyk-Nowak A, Michalek M, Kaluza E, Cepiel A and Paslawska, 2017. Prevalence of arrhythmias in dogs examined between 2008 and 2014. J Vet Res, 61: 103-110

Oyama MA, Fox PR, Rush JE, Rozanski EA and Lesser M, 2008. Clinical utility of serum $\mathrm{N}$-terminal pro-B-type natriuretic peptide concentration for identifying cardiac disease in dogs and assessing 
disease severity. J Am Vet Med Assoc, 232(10): 1496-1503

Oyama MA, 2009. Cardiac drugs for treatment of canine heart failure. NAVC Clinician's Brief, pp756-759, Retrieved from https:// repository.upenn.edu/vet_papers/1

Pedersen HD, Nørby B and Lorentzen KA, 1996. Echocardiographic study of mitral valve prolapse in Dachshunds. J Vet Med A, 43(1 10): 103-110

Pereira L, Larsson MHMA, Neto ML and de Brito FS, 2004. Cardiomyopathy of the English Cocker Spaniel: clinical, electrocardiographic, radiographic and echocardiographic aspect. Cienc Rural, 34(2): 419-424

Petchdee S and Sompeewong S, 2016. Intravenous administration of puppy deciduous teeth stem cells in degenerative valve disease. Vet World, 9(12): 1429-1434

Rao VV, Vasu K and Prathaban S, 2008. Echocardiographic evaluation of dilated cardiomyopathy in dogs. Indian Vet $\mathbf{J}$, 85(11): $82-84$

Rajewski G. 2017. Stem cell studies of congestive heart failure in dogs could benefit humans, too. Cardiology, Tufts University, USA

Reetu, 2018. Diagnostic approaches to canine cardiac diseases with special reference to Doppler ultrasound. Ph. D. thesis, IVRI, Izatnagar

Reetu, Hoque M, Saxena AC, Pawde AM,Verma NK et al., 2017. Incidence of cardiac diseases in dogs: A retrospective study. Indian J Vet Med, 37(1\&2): 64-67
Rishniw M and Erb HN, 2000. Evaluation of four 2 dimensional echocardiographic methods of assessing left atrial size in dogs. J Vet Intern Med, 14(4): 429-435

Rush JE, 2002. Chronic valvular heart disease in dogs. Proceedings from: $26^{\text {th }}$ Annual Waltham Diets/OSU Symposium for the Treatment of Small Animal Cardiology, October, 19-20, 2002

Saxena AC, 2008. Studies on the measurement of reference ranges of echocardiographic indices and related parameters in Indian Spitz breed of dogs. M.V.Sc thesis, IVRI, Izatnagar

Saxena AC, 2014. Studies on regenerative potential of mesenchymal stem cells in cryoinfarcted myocardium in rabbit model. $\mathrm{Ph}$. D. thesis, IVRI, Izatnagar

Schober KE and Fuentes VL, 2001. Effects of age, body weight, and heart rate on transmitral and pulmonary venous flow in clinically normal dogs. Am J Vet Res, 62(9): 1447-1454

Schober KE, Hart TM, Stern JA, Li X, Samii VF et al., 2010). Detection of congestive heart failure in $\operatorname{dog} s$ by Doppler echocardiography. J Vet Intern Med, 24(6): 1358-1368

Serres F, Chetboul V, Tissier R, Sampedrano CC, Gouni V et al., 2007. Chordae tendineae rupture in dogs with degenerative mitral valve disease: prevalence, survival, and prognostic factors (114 cases, 20012006). J Vet Intern Med, 21(2): 258

Shell WE, Kjekshus JK and Sobel BE, 1971. Quantitative assessment of the extent of myocardial infarction in the conscious dog 
by means of analysis of serial changes in serum creatine phosphokinase activity. J Clin Invest 50(12): 2614

Sleeper MM, Henthorn PM and Vijayasarathy HC, 2002. Dilated cardiomyopathy in juvenile portuguese water dogs. J Vet Intern Med, 16: 52-62

Snyder PS, Sato T and Atkins CE, 2005. A comparision of echocardiographic indices of the non racing, healthy Greyhound to reference values from other breeds. Vet Radiol Ultrasound, 36(5): 387-392

Saunders AB, Smith BE, Fosgate GT, Suchodolski JS and Steiner JM, 2009. Cardiac troponin I and C-reactive protein concentrations in dogs with severe pulmonic stenosis before and after balloon valvuloplasty. J Vet Cardiol, 11: 9-16

Taylor DH and Sittnikow KL, 1968. The diagnosis of canine cardiac disease. J Small Anim Pract, 9(12): 589-595

Thomas WP, Gaber CE, Jacobs GJ, Kaplan PM, Lombard CW et al., 1993. Recommendation for standards in transthoracic two-dimensional echocardiography in the dog and cat. J Vet Intern Med, 7: 247-252

Thrall DE, 2007.Veterinary Radiology. $5^{\text {th }}$ edn., Philadelphia, Saunders Elsevier, pp479-485
Thrusfield MV, Aitken CGG and Darker PGG, 1985. Observations on breed and sex in relation to canine heart valve incompetence. J Small Anim Pract, 26(12): 709-717

Tilley LP, 1985. Essentials of canine and feline echocardiography: Interpretation and treatment. $2^{\text {nd }}$ edn., Lea and Febiger, Philedelphia

Tilley LP, Smith Jr FWK, Oyama MA and Sleeper MM, 2008. Manual of canine and feline cardiology. $4^{\text {th }}$ edn., Saunders (Elsevier), St. Louis, Missouri

Vengsarkar SA, 1988. The diagnosis of cardiac diseases in canines. M.V.Sc. thesis, Bombay Veterinary College, Mumbai, India

Visser LC, Im MK, Johnson LR and Stern JA, 2016. Diagnostic value of right pulmonary artery distensibility index in dogs with pulmonary hypertension: comparison with Doppler echocardiographic estimates of pulmonary arterial pressure. $\mathrm{J}$ Vet Intern Med, 30(2): 543-552

Wolfram JA and Donahue JK, 2013. Gene therapy to treat cardiovascular disease. $\mathbf{J}$ Am Heart Assoc, 2(4): e000119, doi: 10.1161/JAHA.113.000119 